\title{
Comparison of the effect of vitamin $E$, vitamin $D$ and ginger on the severity of primary dysmenorrhea: a single-blind clinical trial
}

\author{
Hamideh Pakniat ${ }^{1}$, Venus Chegini', Fatemeh Ranjkesh², Mohammad Ali Hosseini ${ }^{3}$ \\ ${ }^{1}$ Department of Obstetrics and Gynecology, Kowsar Hospital, ${ }^{2}$ Department of Midwifery, Faculty of Nursing and Midwifery, ${ }^{3}$ Student Research \\ Committee, Qazvin University of Medical Sciences, Qazvin, Iran
}

\section{Objective}

Dysmenorrhea is one of the most common problems for women, especially during their reproductive age. Various studies have examined the effects of vitamins $D$ and $E$ and ginger supplements. This study aimed to investigate the individual effects of each of these supplements on dysmenorrhea.

\section{Methods}

This single-blind clinical trial was conducted in 2016 on students aged 18 to 25 years who complained of mild to severe dysmenorrhea. The participants were randomly assigned into 4 groups: vitamin D, vitamin E, ginger, and placebo. The effects of the supplements on the severity of dysmenorrhea were evaluated in 2 successive cycles using the visual analog scale (VAS) and a questionnaire.

\section{Results}

Initially, 240 female students were enrolled in the study; thereafter, 40 students were excluded from the study owing to follow-up loss. The average VAS score for dysmenorrhea in the entire study population was $7.13 \pm 0.80$ before the intervention; the mean VAS score after the first and second months of supplement use was $5.37 \pm 1.51$ and $4.93 \pm 1.48$, respectively. The highest reduction in pain severity was observed in the ginger group ( $F=74.54, P<0.001)$.

\section{Conclusion}

Vitamin $D$, vitamin $E$, and ginger significantly reduced the severity of dysmenorrhea, with ginger having the most significant effect followed by vitamin D and vitamin $\mathrm{E}$. Given the low risk of these supplements, more studies must be conducted on their use as opposed to analgesics.

Keywords: Ginger; Dysmenorrhea; Menstrual cycle; Vitamin E; Vitamin D

\section{Introduction}

Primary dysmenorrhea is painful menstrual cramps with no obvious pelvic pathology, starting at the beginning of menstrual period and lasting for $12-48$ hours. This pelvic pain occurs only in menstruating women and affects their quality of life [1]. The prevalence of dysmenorrhea has been reported to range from $50 \%$ to $90 \%$ in various populations. According to some studies, its prevalence in Iran is approximately $74-90 \%$ [2].

The exact pathogenesis of primary dysmenorrhea is unknown; however, according to some studies, prostaglandins are involved in causing contractions and in primary dysmen- orrhea. Approximately $80 \%$ of affected women experience

Received: 2019.03.13. Revised: 2019.06.08. Accepted: 2019.07.01. Corresponding author: Mohammad Ali Hosseini

Student Research Committee, Qazvin University of Medical Sciences, Shahid Bahonar Boulevard, Qazvin 34199-15315, Iran

E-mail: smahoseini@gmail.com

https://orcid.org/0000-0002-9693-7388

Articles published in Obstet Gynecol Sci are open-access, distributed under the terms of the Creative Commons Attribution Non-Commercial License (http://creativecommons. org/licenses/by-nc/3.0/) which permits unrestricted non-commercial use, distribution, and reproduction in any medium, provided the original work is properly cited.

Copyright $\odot 2019$ Korean Society of Obstetrics and Gynecology 


\section{Obstetrics \& Gynecology Science}

Hamideh Pakniat, et al. Vitamin E, D, ginger and dysmenorrhea

pain relief with the use of prostaglandin inhibitors [3]. Therefore, one major goal of treatment is to reduce the prostaglandin level in the body.

Various approaches and nutritional alternatives have been used to relieve dysmenorrhea, including psychotherapy, transcutaneous electrical nerve stimulation, and administration of nonsteroidal antiinflammatory drugs (NSAIDs), oral contraceptive pills (OCPs), and vitamins [4]. However, the use of NSAIDs and OCPs has limitations, such as patients' lack of response to treatment (with a failure rate of approximately $15 \%)$ and discontinuation owing to side effects or medicine interactions [2]. Therefore, more studies are needed to investigate alternative therapies.

Traditionally, vitamin D has been used to improve dysmenorrhea. The relationship between calcium intake and dysmenorrhea can be ascribed to the function of calcium in reducing contractions. Low levels of calcium intensify uterine spasticity and contractions. Calcium homeostasis is affected by the vitamin $\mathrm{D}$ level; therefore, it can be effective in improving dysmenorrhea [5].

Vitamin $\mathrm{E}$ is also used for improving dysmenorrhea. Reduction of the progesterone level in the luteal phase of the menstrual cycle can trigger enzyme lysis, phospholipid peroxidation, and arachidonic acid production. These changes all lead to increased production of prostaglandins, which will consequently stimulate uterine cramps and contractions $[6,7]$. With its antioxidant properties, vitamin $\mathrm{E}$ reduces phospholipid peroxidation and inhibits the release of arachidonic acid and its conversion to prostaglandins. Therefore, it can play a significant role in relieving the severity of dysmenorrhea [6-10].

Zingiber (ginger) has at least a 2,500-year history in the treatment of many diseases. The use of ginger has long been established for the reduction of pain in dysmenorrhea, osteoarthritis, rheumatoid arthritis, migraine, and gastrointestinal symptoms, such as nausea, vomiting, and diarrhea. It is registered as a herbal medicine with the American Food and Drug Administration [11].

The pain relief mechanism of ginger is not well understood. Nevertheless, evidence suggests that ginger has ingredients (i.e., gingerol and gingerdione) with anti-inflammatory and analgesic effects [12]. Furthermore, laboratory studies suggest that ginger is involved in the relief of dysmenorrhea through the inhibition of cyclooxygenase, which consequently suppresses prostaglandins and leukotrienes.

The present study was performed given the high preva- lence of dysmenorrhea among young women along with its negative impact on their quality of life, career, and education and the economic burden related to the frequent absence from work; this study aimed to compare the efficacy of vitamin $D$, vitamin $E$, and ginger in relieving and improving dysmenorrhea.

\section{Materials and methods}

This single-blind clinical trial was conducted in 2016 on medical students aged $18-25$ years.

Patients with moderate to severe dysmenorrhea, regular menstrual cycles of 21-35 days, menstrual period of 3-7 days, and otherwise healthy conditions were selected for the study. At the time of enrolment, information, including age, weight, height, field of study, occupation, parenting education, exercise, date of first menarche, duration of menstrual cycle, duration of menstrual flow, and pain severity, was recorded. Pre-intervention pain severity was determined using the verbal multidimensional scoring system [13]. In this system, the severity of dysmenorrhea was classified according to a 4-level scale. The subjects who did not have any history of painful menstruation were categorized under level 0 and those with painful menstruation who rarely needed analgesics under level 1. These 2 groups were later excluded from the study. The women whose daily life was affected by dysmenorrhea and symptoms were improved by taking NSAIDs were categorized under level 2 (i.e., moderate dysmenorrhea) and those whose daily life was seriously affected by dysmenorrhea and rarely responded to any NSAIDs under level 3. The latter had other symptoms, such as headache, nausea, fatigue, vomiting, and diarrhea. Only those with moderate to severe dysmenorrhea were finally included in the study. Women with underlying diseases, e.g., endometriosis, fibroids (myomas), adenomyosis, endometrial polyps, and pelvic inflammatory disease, those who use an intrauterine contraceptive device, pregnant women, those taking OCPs, those with mild dysmenorrhea, and those with allergy to synthetic or herbal medicines were excluded.

To demonstrate a significant difference among ginger, vitamin $D$, vitamin $E$, and placebo using the pain severity test, the estimated sample size was calculated to be at least 50 individuals per group based on previous studies $[12,14,15]$. A study with such a sample size would have a power of 


\title{
Obstetrics \& Gynecology Science
}

\author{
Vol. 62, No. 6, 2019
}

$80 \%$ at a significance level of 0.05 . The permuted block randomization method was used to assign the subjects into the 4 groups randomly. Considering the ethical issues regarding preventing the use of analgesics in the women with moderate to severe dysmenorrhea, a specific type of analgesic was selected for all 4 groups. The following were prescribed to the 4 groups: ginger group: ginger capsules (Zingiber officinale) 500 mg per day (Vomigone; Dineh Co., Tehran, Iran); each coated capsule contains $470 \pm 30 \mathrm{mg}$ ginger rhizome powder standardized as $5 \mathrm{mg}$ essential oil and $3 \mathrm{mg}$ total polyphenols, and mefenamic acid $250 \mathrm{mg}$ capsule (Ponstan; Razak Co., Tehran, Iran) twice per day was also administered; placebo group: placebo capsule (Dineh Co.) with mefenamic acid 250 mg capsule twice per day; vitamin E group: 100unit vitamin E capsule as soft gelatin capsule (E-VIGEL; Dana Co., Hamadan, Iran) with mefenamic acid $250 \mathrm{mg}$ capsule twice per day; and vitamin $D$ group: 1,000 mg vitamin $D$ tablet (D-VIGEL; Dana Co.) with mefenamic acid 500 mg capsule in 2 divided doses. The supplements were packaged in similar envelopes. All subjects were advised to take either of 2 medicines (tablet or capsule) and continue consumption up to 3 days after the onset of menstrual flow (total of 5 days). The severity of dysmenorrhea was evaluated after the end of menstruation. The effect of the supplements on dysmenorrhea was analyzed after the treatment period using the visual analog scale (VAS) [16]. The patients were instructed to mark the severity of their pain on a $10-\mathrm{cm}$ ruler. The beginning and end of the ruler represent no pain and severe pain, respectively. Therefore, the patients were divided according to their severity of pain: painless (score, 0), mild (score, 1-3), moderate (score, 4-7), and severe (score, 8-10) based on 2 sequential cycles of review.

Finally, a questionnaire was used, and data were analyzed using the SPSS software (IBM Corp., Armonk, NY, USA); analysis of variance (ANOVA) and the $\chi^{2}$ and Tukey tests were used to determine the difference in the patients' general characteristics, severity of disease or pain, and satisfaction with the intervention. The significance level was set at 0.05 .

\section{Results}

Initially, 240 female students were enrolled in the study; thereafter, 40 students were excluded from the study owing to follow-up loss. Finally, 200 subjects were analyzed (Fig. 1). The average age of the participants was $22.44 \pm 1.92$ years; the average BMI was $21.62 \pm 3.15 \mathrm{~kg} / \mathrm{m}^{2}$; the average age at menarche was $12.55 \pm 1.00$ years; the average duration of menstrual cycle was $25.30 \pm 1.07$ days; the average duration of menstruation was $5.2 \pm 0.95$ days; the average duration of dysmenorrhea was $2.6 \pm 0.89$ days; The average VAS score for dysmenorrhea in the entire study population was $7.13 \pm 0.80$ before the intervention; the mean VAS score after the first and second months of supplement use was $5.37 \pm 1.51$ and $4.93 \pm 1.48$, respectively (Fig. 2 ). The demographic characteristics and menstrual profile of the subjects are presented in Table 1.

As shown in Table 1, there was no significant difference in

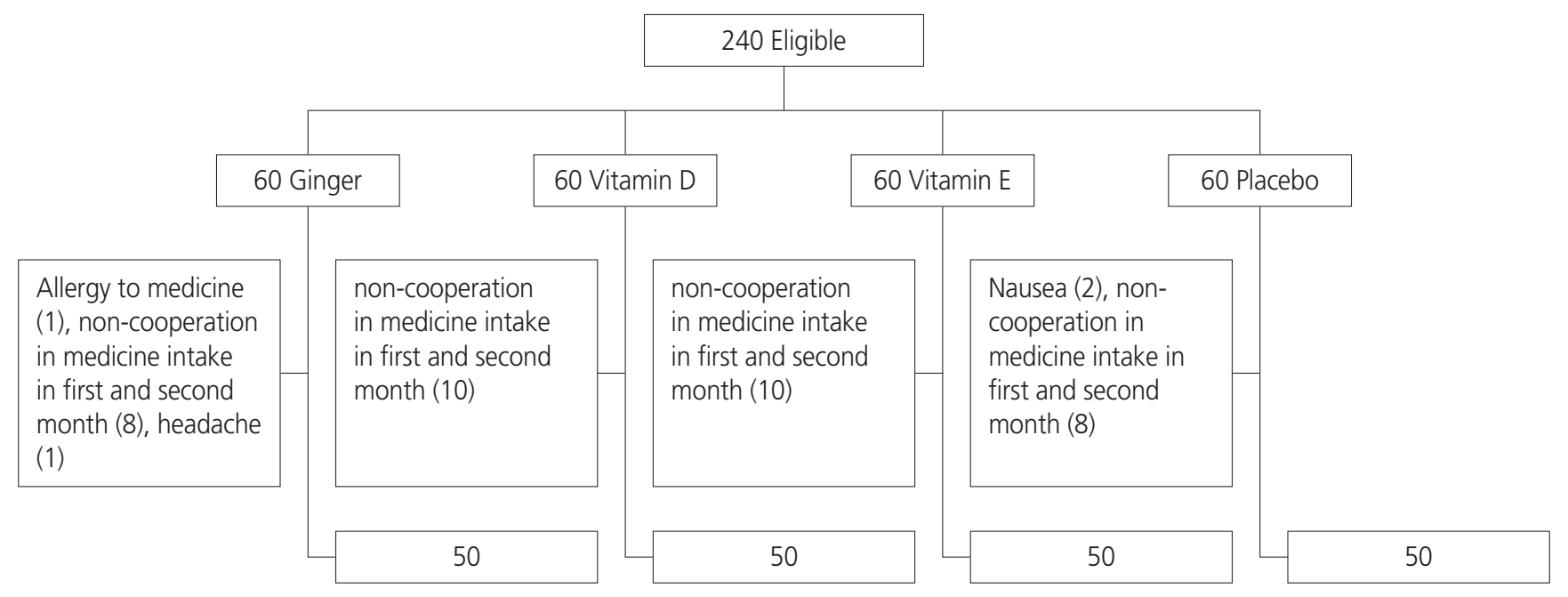

Fig. 1. patients allocation. 


\section{Obstetrics \& Gynecology Science}

Hamideh Pakniat, et al. Vitamin E, D, ginger and dysmenorrhea

the average demographic characteristics and menstrual data among the 4 groups.

The severity of dysmenorrhea was compared among the 4 groups before and after the first and second months of in-

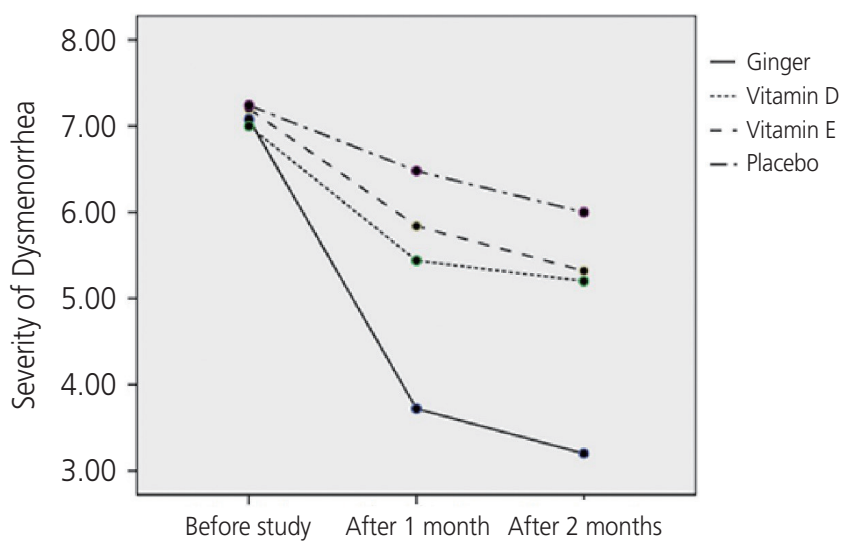

Fig. 2. The average pain severity in 3 periods in different groups. VAS, visual analog scale. tervention using the Kruskal-Wallis test. The analysis showed that there was no significant difference among the groups before the intervention ( $P=0.52)$. However, there was a significant difference in the first and second months after taking the supplements ( $P<0.001$ in both months). Repeatedmeasures ANOVA revealed that the severity of dysmenorrhea had the highest decrease in the ginger group ( $F=74.54$, $P<0.001$ ) (Table 2). The mean reduction in pain severity (i.e., VAS score) after the first and second months of intervention was $3.36,1.56$, and 1.36 and $3.88,1.8$, and 1.88 in the ginger, vitamin $\mathrm{D}$, and vitamin $\mathrm{E}$ groups, respectively $(P<0.05)$ (Table 2).

The side effects that were taken into account included nausea, vomiting, allergy, headache, and heartburn, which had no significant difference among the groups. Mild nausea was observed in 2 women in the placebo group and mild headache in 1 woman and sensitivity to the medicine in another woman in the ginger group.

Table 1. Demographic characteristics and menstrual profile of the subjects (analysis of variance)

\begin{tabular}{lccccc}
\hline Variable & Ginger & Vitamin D & Vitamin E & Placebo & $P$-value \\
\hline Age & $22.8 \pm 1.8$ & $22.7 \pm 1.5$ & $22.3 \pm 1.4$ & $22.3 \pm 1.6$ & 0.586 \\
$\mathrm{BMI}\left(\mathrm{kg} / \mathrm{m}^{2}\right)$ & $21.47 \pm 2.41$ & $21.78 \pm 3.42$ & $21.32 \pm 3.67$ & $21.91 \pm 3.03$ & 0.770 \\
Menarche age (yr) & $12.78 \pm 0.81$ & $12.78 \pm 1.07$ & $12.90 \pm 0.88$ & $12.96 \pm 1.21$ & 0.755 \\
Duration of menstrual cycle (day) & $25.35 \pm 1.12$ & $25.26 \pm 1.15$ & $25.32 \pm 1.09$ & $25.26 \pm 1.02$ & 0.969 \\
Duration of menstruation (day) & $5.14 \pm 0.83$ & $5.08 \pm 0.92$ & $5.32 \pm 1.26$ & $5.26 \pm 0.72$ & 0.582 \\
Duration of dysmenorrhea (day) & $2.60 \pm 0.75$ & $2.52 \pm 0.81$ & $2.76 \pm 1.07$ & $2.60 \pm 0.90$ & 0.596 \\
\hline
\end{tabular}

Data are shown as mean \pm standard deviation.

BMI, body mass index.

Table 2. Average pain severity before and after the intervention in the 4 groups (visual analog scale [VAS] score)

\begin{tabular}{|c|c|c|c|c|c|}
\hline Variable & Ginger & Vitamin D & Vitamin E & Placebo & $P$-value \\
\hline Pre-intervention & $7.08 \pm 0.8$ & $7.01 \pm 0.11$ & $7.20 \pm 0.64$ & $7.24 \pm 0.56$ & 0.520 \\
\hline First month post-intervention & $3.72 \pm 1.39$ & $5.44 \pm 1.40$ & $5.84 \pm 0.84$ & $6.48 \pm 0.71$ & $<0.001$ \\
\hline Second month post-intervention & $3.20 \pm 1.28$ & $5.20 \pm 1.34$ & $5.32 \pm 0.68$ & $6.0 \pm 0.70$ & $<0.001$ \\
\hline VAS change 1st & $3.36 \pm 1.13^{\mathrm{a}, \mathrm{b} b, \mathrm{c}}$ & $1.56 \pm 0.90^{\mathrm{a}, \mathrm{e})}$ & $1.36 \pm 0.56^{\mathrm{b}), \mathrm{f}}$ & $0.76 \pm 0.84^{(\mathrm{c}, \mathrm{e}, \mathrm{f})}$ & $<0.001$ \\
\hline VAS change 2 nd & $3.88 \pm 1.00^{\mathrm{a}, \mathrm{b}), \mathrm{c})}$ & $1.8 \pm 0.85^{\mathrm{a}, \mathrm{e})}$ & $1.88 \pm 0.59^{b), f)}$ & $1.24 \pm 0.89^{c(,), f)}$ & $<0.001$ \\
\hline
\end{tabular}

VAS change 1st, amount of reduction in the VAS score in the first month compared with that at pre-intervention; VAS change 2nd, amount of reduction in the VAS score in the second month compared with that at pre-intervention.

${ }^{a}$ Significant difference between the ginger and vitamin D groups derived using the Tukey test $(P<0.05)$; ${ }^{b}$ Significant difference between the ginger and vitamin $\mathrm{E}$ groups derived using the Tukey test $(P<0.05)$; ${ }^{\mathrm{c}}$ Significant difference between the ginger and placebo groups derived using the Tukey test $(P<0.05)$; ${ }^{\text {d) }}$ Significant difference between the vitamin $\mathrm{D}$ and vitamin $\mathrm{E}$ groups derived using the Tukey test $(P<0.05)$; ${ }^{\text {e) }}$ Significant difference between the vitamin $\mathrm{D}$ and placebo groups derived using the Tukey test $(P<0.05)$; ${ }^{f}$ Significant difference between the vitamin $\mathrm{E}$ and placebo groups derived using the Tukey test $(P<0.05)$. 


\title{
Obstetrics \& Gynecology Science
}

\author{
Vol. 62, No. 6, 2019
}

\section{Discussion}

Several studies have investigated the effects of vitamins $D$ and $E$ and ginger on primary dysmenorrhea $[2,4,7-$ $9,11,12,14,15,17-28]$. However, this study is the first clinical trial that aimed to compare their cumulative effects on the severity of dysmenorrhea. In this study, all participants were administered with mefenamic acid (analgesic); vitamin D, vita$\min E$, and ginger were separately prescribed to each group. In our analysis, the pain severity decreased in the vitamin $E$, vitamin $D$, and ginger groups, with the highest decrease observed in the ginger group. Our findings are consistent with those of several other studies. Akhlaghi et al. [2] investigated the effect of vitamin $E$ on the severity of primary dysmenorrhea and argued that this vitamin has a significant effect on the severity and duration of dysmenorrhea. Kashanian et al. [22] conducted a single-blind clinical trial on 94 women who received $400 \mathrm{IU}$ vitamin E per day and concluded that vitamin $E$ and placebo can reduce the severity of pain in primary dysmenorrhea; however, vitamin E reduced the pain severity more than did placebo. According to previous studies, pain relief in dysmenorrhea after vitamin E injection was associated with a significant increase in $\beta$-endorphin-like substances. It is believed that the mechanism of pain relief in dysmenorrhea with the use of vitamin $\mathrm{E}$ can be attributed to the increase in $\beta$-endorphins. In 2018, Sadeghi et al. [8] demonstrated that the combination of vitamin $E$ and omega-3 can reduce pain severity in dysmenorrhea. A known mechanism for the reduction of the severity of dysmenorrhea by omega-3 is inhibition of prostaglandin synthesis. They showed that the combination of vitamin E and omega-3 significantly decreased the pain severity in all their groups, with the highest decrease in the omega- 3 and vitamin D groups followed by the vitamin E group.

Many studies demonstrated the effective role of vitamin $D$ in the pain relief of primary dysmenorrhea, which is consistent with our results. In the first clinical trial conducted with a high single dose $(300,000)$ of vitamin Lasco et al. [24] showed that the severity of dysmenorrhea decreased by $41 \%$, and the highest reduction was observed in women who had the highest level of pain severity. In their clinical trial study on 60 women with dysmenorrhea and vitamin $D$ deficiency who were prescribed with a supplement of 50,000 IU vitamin D, Moini et al. [15] found that their intervention group had a significant pain relief compared with their con- trol group after 8 weeks. In their study on 897 individuals in 2018, Bahrami et al. [17] argued that vitamin D supplements significantly reduced the severity of dysmenorrhea as well as premenstrual syndrome (PMS) symptoms.

Although the exact mechanism of pain relief with the use of vitamin D in dysmenorrhea is not yet fully understood, studies demonstrated its important role in other menstrualrelated pains, such as PMS, fibromyalgia, and endometriosis. In a cross-sectional study conducted in 2010, women who received $\geq 100 \mathrm{IU}$ vitamin $D$ from food sources had $70 \%$ less symptoms of PMS than those who received $<100 \mathrm{IU}$ vitamin D. Moreover, several studies investigated the role of ginger in reducing the severity of primary dysmenorrhea. In 2009, Ozgoli et al. [26] conducted a study on 150 students and argued that ginger reduced the severity of dysmenorrhea in the same manner as ibuprofen and mefenamic acid without a significant difference. With an efficiency of $80 \%$, mefenamic acid and ibuprofen are the main medicine options for dysmenorrhea. In their study on 120 students with moderate to severe dysmenorrhea, Rahnama et al. [12] investigated the use of ginger supplements in 2 groups (treatment and placebo) with 2 different protocols of $500 \mathrm{mg}$ capsules for 5 and 3 days. They reported that the duration of dysmenorrhea was significantly shorter in the treatment group. The severity of dysmenorrhea decreased in both groups, and the first group had significantly lower levels of pain.

NSAIDs are effective in the treatment of dysmenorrhea but are associated with considerable morbidity and mortality. Therefore, ginger is a good treatment option to reduce the severity of dysmenorrhea.

In a study conducted in 2013 on the administration of ginger supplements at a dose of $500 \mathrm{mg} 3$ times a day for 3 days, it was reported that $82.85 \%$ of patients experienced pain relief for dysmenorrhea after the subsequent menstrual period. Many studies investigated the individual effects of vitamin $E$, vitamin $D$, and ginger on the severity of dysmenorrhea. However, none of these studies compared their total effects on dysmenorrhea. In our study, the highest reduction in pain severity was observed in the ginger group followed by the vitamin $D$, vitamin $E$, and placebo groups.

Although NSAIDs are the medicine of choice for the treatment of dysmenorrhea, numerous complications are associated with their use; thus, based on our results, vitamin $D$, vitamin $E$, and especially ginger can be used as alternatives in reducing the severity of dysmenorrhea. More extensive stud- 


\section{Obstetrics \& Gynecology Science}

Hamideh Pakniat, et al. Vitamin E, D, ginger and dysmenorrhea

ies must be conducted to prove this finding. The limitations of this study included the lack of cooperation by some students and their consequent exclusion. Furthermore, owing to ethical issues, all 3 intervention groups were administered with analgesics, and it was not possible to investigate the individual effect of ginger, vitamin $E$, and vitamin $D$ on the severity of dysmenorrhea; therefore, we only compared their effects on dysmenorrhea.

In conclusion, ginger was the most effective supplement among the supplements administered. Conversely, vitamin $\mathrm{D}$ appeared to be more effective than vitamin $\mathrm{E}$ in relieving dysmenorrhea.

\section{Conflict of interest}

No potential conflict of interest relevant to this article was reported.

\section{Ethical approval}

This study was approved by the Ethics Committee of Qazvin University of Medical Sciences (IR.QUMS.REC.1395.205) and registered at IRCT (IRCT20120104008611N7).

\section{Patient consent}

Informed consent was obtained from all students.

\section{References}

1. Treede RD, Rief W, Barke A, Aziz Q, Bennett MI, Benoliel $R$, et al. A classification of chronic pain for ICD-11. Pain 2015;156:1003-7.

2. Akhlaghi F, Zyrak N, Nazemian S. Effect of vitamin E on primary dysmenorrhea. Hayat 2009;15:82.

3. Penland JG, Johnson PE. Dietary calcium and manganese effects on menstrual cycle symptoms. Am J Obstet Gynecol 1993;168:1417-23.

4. Abdul-Razzak KK, Obeidat BA, Al-Farras MI, Dauod AS. Vitamin D and PTH status among adolescent and young females with severe dysmenorrhea. J Pediatr Adolesc
Gynecol 2014;27:78-82.

5. Alexandrovich I, Rakovitskaya O, Kolmo E, Sidorova T, Shushunov S. The effect of fennel (Foeniculum Vulgare) seed oil emulsion in infantile colic: a randomized, placebo-controlled study. Altern Ther Health Med 2003;9:5861.

6. Ostad SN, Soodi M, Shariffzadeh M, Khorshidi N, Marzban $\mathrm{H}$. The effect of fennel essential oil on uterine contraction as a model for dysmenorrhea, pharmacology and toxicology study. J Ethnopharmacol 2001;76:299304.

7. Kucukceran H, Ozdemir O, Kiral S, Berker DS, Kahveci R, Ozkara A, et al. The impact of circulating 25-hydroxyvitamin and oral cholecalciferol treatment on menstrual pain in dysmenorrheic patients. Gynecol Endocrinol 2019;35:53-7.

8. Sadeghi N, Paknezhad F, Rashidi Nooshabadi M, Kavianpour M, Jafari Rad S, Khadem Haghighian H. Vitamin E and fish oil, separately or in combination, on treatment of primary dysmenorrhea: a double-blind, randomized clinical trial. Gynecol Endocrinol 2018;34:804-8.

9. Karacin O, Mutlu I, Kose M, Celik F, Kanat-Pektas M, Yilmazer M. Serum vitamin D concentrations in young Turkish women with primary dysmenorrhea: a randomized controlled study. Taiwan J Obstet Gynecol 2018;57:58-63.

10. Saboori S, Falahi E, Birjandi M, Hesami S, Veisi F, Hosseini MA. Could co-administration of vitamins $E$ and $C$ increase total antioxidant capacity? A systematic review and meta-analysis. Curr Top Nutraceutical Res 2018;16:253.

11. Daily JW, Zhang $X$, Kim DS, Park S. Efficacy of ginger for alleviating the symptoms of primary dysmenorrhea: a systematic review and meta-analysis of randomized clinical trials. Pain Med 2015;16:2243-55.

12. Rahnama P, Montazeri A, Huseini HF, Kianbakht S, Naseri $M$. Effect of Zingiber officinale R. rhizomes (ginger) on pain relief in primary dysmenorrhea: a placebo randomized trial. BMC Complement Altern Med 2012;12:92.

13. Wall P, Melzack R. Text book of pain. 5th ed. London: Churchill Living Stone; 1999.

14. Ziaei S, Faghihzadeh S, Sohrabvand F, Lamyian M, Emamgholy $\mathrm{T}$. A randomised placebo-controlled trial to determine the effect of vitamin $E$ in treatment of primary dysmenorrhoea. BJOG 2001;108:1181-3. 


\section{Obstetrics \& Gynecology Science}

Vol. 62, No. 6, 2019

15. Moini A, Ebrahimi T, Shirzad N, Hosseini R, Radfar M, Bandarian $F$, et al. The effect of vitamin $D$ on primary dysmenorrhea with vitamin $D$ deficiency: a randomized double-blind controlled clinical trial. Gynecol Endocrinol 2016;32:502-5.

16. Kim YI, Nam SG, Hong ST, Kang KS, Park W. Comparison of visual analogue scale, categorical scale and satisfaction for postoperative pain. J Korean Pain Soc 2001;14:156-63.

17. Bahrami A, Avan A, Sadeghnia HR, Esmaeili H, Tayefi M, Ghasemi $F$, et al. High dose vitamin D supplementation can improve menstrual problems, dysmenorrhea, and premenstrual syndrome in adolescents. Gynecol Endocrinol 2018;34:659-63.

18. Chen CX, Barrett B, Kwekkeboom KL. Efficacy of oral ginger (zingiber officinale) for dysmenorrhea: a systematic review and meta-analysis. Evid Based Complement Alternat Med 2016;2016:6295737.

19. Shirvani MA, Motahari-Tabari N, Alipour A. The effect of mefenamic acid and ginger on pain relief in primary dysmenorrhea: a randomized clinical trial. Arch Gynecol Obstet 2015;291:1277-81.

20. Kashefi F, Khajehei M, Tabatabaeichehr M, Alavinia $M$, Asili J. Comparison of the effect of ginger and zinc sulfate on primary dysmenorrhea: a placebo-controlled randomized trial. Pain Manag Nurs 2014;15:826-33.

21. Jenabi $E$. The effect of ginger for relieving of primary dysmenorrhoea. J Pak Med Assoc 2013;63:8-10.

22. Kashanian M, Lakeh MM, Ghasemi A, Noori S. Evalua- tion of the effect of vitamin $E$ on pelvic pain reduction in women suffering from primary dysmenorrhea. J Reprod Med 2013;58:34-8.

23. Bertone-Johnson ER, Manson JE. Vitamin D for menstrual and pain-related disorders in women: comment on "improvement of primary dysmenorrhea caused by a single oral dose of vitamin D". Arch Intern Med 2012;172:367-9.

24. Lasco A, Catalano A, Benvenga S. Improvement of primary dysmenorrhea caused by a single oral dose of vitamin D: results of a randomized, double-blind, placebocontrolled study. Arch Intern Med 2012;172:366-7.

25. Sun LH, Ge JJ, Yang JJ, She YF, Li WL, Li XH, et al. Randomized controlled clinical study on ginger-partitioned moxibustion for patients with cold-damp stagnation type primary dysmenorrhea. Zhen Ci Yan Jiu 2009;34:398402.

26. Ozgoli G, Goli M, Moattar F. Comparison of effects of ginger, mefenamic acid, and ibuprofen on pain in women with primary dysmenorrhea. J Altern Complement Med 2009;15:129-32.

27. Bertone-Johnson ER, Chocano-Bedoya PO, Zagarins SE, Micka $A E$, Ronnenberg AG. Dietary vitamin D intake, 25-hydroxyvitamin D3 levels and premenstrual syndrome in a college-aged population. J Steroid Biochem Mol Biol 2010;121:434-7.

28. Krishnan AV, Feldman D. Mechanisms of the anti-cancer and anti-inflammatory actions of vitamin D. Annu Rev Pharmacol Toxicol 2011;51:311-36. 